RUNNING HEAD: DATA LINKAGE CONSENT FOR DIFFERENT DATA

DOMAINS

\title{
Consent to Data Linkage for Different Data Domains - The Role of Question Order, Question Wording, and Incentives
}

\author{
Christoph Beuthner ${ }^{\mathrm{a}}$, Florian Keusch ${ }^{\mathrm{b}}$, Henning Silber ${ }^{\mathrm{a}}$, \\ Bernd Weiß $\beta^{\mathrm{a}}$, and Jette Schröder ${ }^{\mathrm{a}}$ \\ a GESIS - Leibniz Institute for the Social Sciences, Mannheim, Germany \\ b University of Mannheim, Mannheim, Germany
}

Keywords: consent, data linkage, online surveys, experiment

Corresponding author: Christoph Beuthner, GESIS -Leibniz Institute for the Social Sciences, christoph.beuthner@googlemail.com, B6,4-5, 68159 Mannheim, Germany

Recommended citation of this preprint:

Beuthner, C., Keusch, F., Silber, H., Weiß, B., \& Schröder, J. (2022). Consent to Data

Linkage for Different Data Domains - The Role of Question Order, Question Wording, and Incentives. SocArXiv, Available at: https://doi.org/10.31235/osf.io/qh93g 


\begin{abstract}
As our modern world has become increasingly digitalized, various types of data from different data domains are available that can enrich survey data. To link survey data to other sources, consent from the survey respondents is required. This article compares consent to data linkage requests for seven data domains: administrative data, smartphone usage data, bank data, biomarkers, Facebook data, health insurance data, and sensor data. We experimentally explore three factors of interest to survey designers seeking to maximize consent rates: consent question order, consent question wording, and incentives. The results of the study using a German online sample $(n=3,374)$ show that survey respondents have a relatively high probability of consent to share smartphone usage data, Facebook data, and biomarkers, while they are least likely to share their bank data in a survey. Of the three experimental factors, only the consent question order affected consent rates significantly. Additionally, the study investigated the interactions between the three experimental manipulations and the seven data domains, of which only the interaction between the data domains and the consent question order showed a consistent significant effect.
\end{abstract}




\section{Introduction}

With the help of emerging digital technologies, the ability to easily record and process information on people's everyday life offers new possibilities for researchers (Link et al., 2014). Linking additional data with traditional survey data provides an opportunity for survey methodology, a field that permanently tries to reduce survey costs and measurement error. Data linkage could help increase data quality by delivering more reliable data and substituting missing data and therefore increase the value of datasets. As surveys are also used in an increasingly interdisciplinary environment and across different fields of science, including public health, economics, and education, the linkage of additional data might help researchers to generate new knowledge by enriching datasets with information not obtainable from surveys (e.g., linking medical records to self-assessments). Consequently, some studies already exist that link survey data to additional data sources, such as biomarkers (e.g., Avendano et al., 2011; McFall et al., 2014) and administrative data (e.g., Baker et al., 2000; Christoph et al., 2008).

However, scientists cannot simply link additional data but have an ethical and legal obligation to obtain respondents' consent before implementing data linkage procedures. As some data domains (e.g., financial data) are often seen as sensitive by respondents (Walzenbach et al., 2021), asking for and obtaining consent to link these data can be rather difficult. While various factors can influence consent rates, only some can be manipulated by researchers, including incentives, question wording, and the position of the consent request in the questionnaire (Keusch et al., 2019; Revilla et al., 2018; Sakshaug et al., 2012; Sakshaug et al., 2013). So far, only a few studies have compared consent decisions with respect to different data domains (Revilla et al., 2018; Wenz et al., 2019). By investigating contextual factors and different data domains in one study, we will be able to give practical advice to practitioners on how to increase consent rates for data linkage requests in surveys. 
In this study, we investigate respondents' willingness to consent to the linkage of data from various data domains, and we experimentally test the influence of contextual factors that can be optimized to achieve higher consent rates. In particular, we are interested in answering the following research questions: (1) Do consent rates for data linkage differ by data domain? (2) How do contextual factors (i.e., question wording, incentives, and question order) influence consent rates? (3) How do data domains and contextual factors interact with respect to willingness to share additional data?

In the next section of this paper, we discuss previous research on data linkage and develop our research hypotheses. Afterward, we describe the methods used in our study and present our results. Finally, we discuss the theoretical and practical implications of our findings.

\section{Background}

With their consent to data linkage, survey respondents allow the researcher further access to additional personal information. Thus, the act of consenting to data linkage is always associated with a certain amount of previously agreed privacy intrusions (Martin \& Shilton, 2016; Nissenbaum, 2018, 2019). As the perceived sensitivity of data domains might differ, consent rates between data domains might differ. However, researchers can try to increase consent rates by varying certain methodological elements within the consent request(s). In the following, we provide an overview of how the consent decision can differ by data domain and which measures researchers can implement to potentially increase consent rates.

\subsection{Do consent rates for data linkage differ by data domain?}

As data domains might vary in sensitivity, allowing access to them might be associated to higher or lower costs for respondents. Only a few studies confront survey respondents with consent requests regarding different tasks or data domains, which can largely vary regarding the task difficulty and related burden for respondents (Silber et al., 2021). With respect to 
comparing different data domains, Revilla et al. (2018) conducted a study where Spanish respondents in a nonprobability online panel were given a list of 20 different tasks that went beyond responding to a web survey. Consent rates ranged from $74 \%$ for receiving a product at home and testing it to $6 \%$ for letting respondents' children wear a small device that delivers real-time information about the child's stress levels. The results show that respondents were more likely to accept additional tasks if they were able to report the information themselves (e.g., provide a self-report of blood cholesterol levels) compared to when data was shared automatically (e.g., share GPS location via smartphone automatically). Respondents also were less likely to consent when they were asked for measures that allow drawing conclusions about their behavior (e.g., share all information on Facebook profile) compared to other tasks. Wenz et al. (2019) used the Understanding Society Innovation Panel in the UK to ask smartphone respondents for their willingness to perform additional tasks with their smartphones. $65 \%$ of the participants stated that they were willing to take photos or scan barcodes, $61 \%$ would use the built-in accelerometer to record movements, $39 \%$ would share their location via GPS, and $28 \%$ would install an app that tracks their phone usage anonymously.

While no study compared the different data domains that we include in our study, previous studies showed that different consent requests indeed yielded different consent rates. This might be because survey respondents consider data from some domains more sensitive than others and thus are less willing to share this information. Therefore, we assume that a respondent's willingness to consent might differ between different data domains.

\section{H1. Consent rates differ between data domains.}




\subsection{How do contextual factors (i.e., question order, consent question wording and incentives) influence consent rates?}

\subsubsection{Consent question order effect}

Linking several data domains to survey data in one study can help to explore research questions from various angles. This can be necessary in interdisciplinary contexts, for example, when assessing health risks using both information from biomarkers and fitness apps. Nevertheless, research on order effects deriving from multiple requests is rare. To our knowledge, only one study has yet explicitly investigated consent question order effects. Walzenbach et al. (2021) conducted two experiments in two surveys using an access panel in the UK comparing five different data domains. In the first experiment, they tested two question orders (starting with the most sensitive data domain (health data) vs. starting with the least sensitive data domain (tax data)). They found that asking a less sensitive consent request first yields higher average consent rates. Additionally, they could not find an increase or decrease in consent rates associated with question order of the following questions. However, the effects found in the first study could not be replicated in the second study.

Research conducted by Keusch et al. (2019) can deliver further evidence on consent question order effects. In a vignette experiment, web survey respondents had to rate their willingness to participate in studies that involved installing a research app to their smartphone that passively collected data about smartphone usage and geolocation. Respondents received eight study descriptions that experimentally varied several features of the study (e.g., sponsor, incentive, length of data collection period). The stated willingness of respondents to participate in the described study was significantly higher for the first vignette seen compared to all the other seven vignettes regardless of the study description. 
Another study by Sakshaug et al. (2019) focused on the position of a single consent request within the questionnaire. The study showed that a consent request placed at the beginning of the survey increased consent rates by 15.5 percentage points in a telephone sample and by 11.6 percentage points in a web sample, compared to when the question was placed at the end.

Based on these earlier findings, we assume that every additional consent request asked in a survey is associated with additional costs. Thus, respondents should be more likely to consent to earlier consent requests and be less likely to consent to later ones. We hypothesize that a ceiling effect could decrease consent probability for later consent requests drastically (Wang et al., 2008) .

H2.1 The earlier a respondent is confronted with a data linkage request in the sequence of consent questions, the higher the likelihood to consent.

\subsubsection{Consent question wording}

Going beyond research that compared consent rates for different data domains, there is a growing body of literature that investigates how consent rates to data linkage can be increased. In particular for cases when linkage requests pertain to data domains that are perceived as sensitive by many respondents, for example, financial information or information about stigmatized social behavior, the wording of the request seems especially important (Tourangeau \& Yan, 2007). However, the empirical evidence on how much influence question wording actually has on the consent decision is mixed. Pascale (2011) found no significant differences in data linkage consent rates for administrative records in a telephone survey between the stated benefits of reduced costs, reduced time, and better data accuracy. Similarly, Sakshaug et al. (2013) found no differences in consent rates between a neutral and a time savings framing when conducting an experiment in a telephone survey. 
However, in another experimental study, Sakshaug and Kreuter (2014) found a 6 percentage points higher consent rate for linking the web survey responses to administrative records if a time saving framing was used compared to a neutral framing. In a telephone survey, Kreuter et al. (2015) tested a gain against a loss framing using an experimental setup. The gain framing stated that the information provided by the respondent would gain value if the respondent consented to data linkage, whereas the loss framing stated that the information would lose value. They found consent rates to be 10 percentage points higher for the loss than for the gain framing. In another study by Struminskaya et al. (2021), the researchers compared a neutral consent request against one emphasizing time savings arising from the linkage of sensor data using the Dutch LISS panel. However, they did not find a significant effect of the framing.

Using beneficial wording in a consent request can help to balance out privacy costs by associating a benefit (e.g., time savings or added scientific value) with the act of data sharing. In that way, a beneficial statement can help reduce costs arising from sharing information. Therefore, we assume that consent rates can be increased by accompanying the request with a statement that emphasizes specific benefits when consenting.

H2.2 When presented with a stated non-monetary benefit together with the consent request, respondents are more likely to give consent than when the benefit is not stated.

\subsubsection{Incentive}

An incentive might be an efficient way to motivate respondents to give linkage consent. The most straightforward way to do so is to link the consent decision to a financial incentive. With respect to survey participation, incentives have been successfully used to increase response rates and data quality (Singer \& Ye, 2013). The influence of consent-related incentives, however, has not been studied exhaustively. Jäckle et al. (2017) found no significant 
difference between incentives of two and six British Pounds for downloading a spending app in the UK Understanding Society Innovation Panel. In their experimental vignette study, Keusch et al. (2019) found that the willingness to download a research app that would passively collect sensor and log file data from participants' smartphones increased by 18,19 , or 26 percentage points, respectively, when a 10 Euro incentive for downloading the app, a 10 Euro incentive for leaving the app installed until the end of the field period, or when both an incentive for downloading and at the end of the field period were promised, compared to when no incentives were announced.

We expect to find a similar beneficial effect by providing a financial incentive to respondents for making a consent decision.

H2.3 When promised a financial incentive, respondents are more likely to give consent than when no incentive is given.

\subsection{How do data domains and contextual factors interact with respect to willingness to share additional data?}

We assume that the mechanisms of question order, question wording, and incentive influence consent to all seven data domains. However, it is possible that the size of the effect differs between domains. For some data domains, where the perceived costs of data linkage for the respondents are relatively high, the beneficial effects of the contextual factors might be relatively low, as question order, question wording, and incentive might not provide enough of a push for people to give consent. At the same time, for data domains where the perceived costs of data linkage are rather low, and the baseline consent rates are relatively high, the contextual factors might not have a strong additional effect either. As we do not know which consent decisions are more or less costly for respondents, we will not formulate any interaction hypotheses but instead, conduct exploratory analyses. 


\section{Methods}

To study our research questions and test the stated hypotheses, we gathered data from a web survey conducted between July 15 and August 31, 2018. Respondents were recruited from a German nonprobability online access panel. Quotas according to the general population of Germany were set for gender, education, age, and federal state.

In addition to the experimental set-up that allows us to test our hypotheses, the questionnaire also included questions and experiments on other topics (e.g., concerning the device used to respond to the survey, misreporting, attentiveness, and social networks). For example, before receiving the invitation, panel members were randomly allocated to a desktop/laptop computer group and a smartphone group, and in the invitation, panel members were instructed to complete the survey on the assigned device. Violations of the requirement were checked by asking the respondent for the device they used and by analyzing the user agent string. Respondents violating the device assignment were screened out. While this experiment is not the main focus of this study, we do control for the device as part of our analyses. Additionally, questions regarding trust, attitudes, smartphone usage, sociodemographics, and other variables were included. Respondents had the possibility to proceed without answering a survey question but could not to go back in the questionnaire. The questionnaire was optimized for smartphones, meaning that questions and question formats were optimized to be displayed on smaller screens. For example, larger grids were split and presented as individual questions on subsequent screens so that the questionnaire was shown in a similar design on both desktop/laptop computers and mobile devices.

A total of 50,063 panel members were invited of which 6,750 opened the online questionnaire by clicking on the invitation link. 2,838 or $42 \%$ of the panel members who started the survey were screened out because they used a device to which they were not 
assigned, and $538(8 \%)$ broke off the survey before the last question. The final sample consists of 3,374 completed interviews, of which 1,826 completed the survey on a desktop/laptop computer and 1,548 using a smartphone. The median response time for completing the questionnaire was 29 minutes and 36 seconds.

In this paper, we focus on the consent to data linkage module of our questionnaire (for an overview of the question sequence in this module, see Figure 1). The module started with an introductory page, providing information about data protection, and preparing respondents for the consent to data linkage part. At this point, respondents were randomly assigned to one of three different framing conditions. Independent of the framing manipulation, all respondents were also randomly assigned to receive information about an additional incentive or not (the exact wording of the introductory page can be found in Online Appendix A). One the next seven pages, we asked a sequence of seven consecutive consent requests referring to data linkage regarding the seven data domains, which were shown on separate pages and ordered randomly for each respondent to allow experimental comparison. All respondents who consented to the linkage of Facebook data received a page delivering detailed instructions for the actual data linkage after replying to all seven requests. ${ }^{1}$ The remaining respondents were shown a debriefing page, thanking them for their willingness to share data and informing them that no additional data would be gathered. If respondents declined the consent to certain domains, they were asked to explain their decision. Similarly, if respondents consented to the linkage of Facebook data, they were asked to provide a reason for why they did so. ${ }^{2}$ Finally, all respondents were asked if they participated in a survey asking for data linkage of one of our domains before. ${ }^{3}$ The wording of all instructions and

\footnotetext{
${ }^{1}$ The results of the data linkage procedure for Facebook data are not discussed in this paper.

${ }^{2}$ The results of both questions are not discussed in this paper.

${ }^{3}$ This measurement is not included in the analyses because the number of respondents who were confronted with requests for additional data was very small ( $\mathrm{n}>367$ across all data domains combined).
} 
questions can be found in Online Appendix B.

[Figure 1 about here.]

\subsection{Measures}

Summary statistics for all variables can be found in Table 1.

\subsubsection{Experimental manipulations}

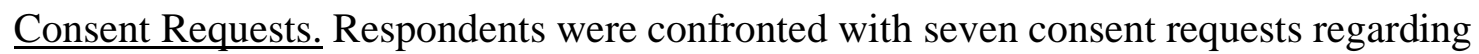
different data domains in random order resulting in 5,040 unique possible question sequences. All of the requests named the type of data which would be collected and gave examples of why these data are of interest (e.g., the number of friends for Facebook data to investigate social interactions on social media platforms). These seven requests, which were placed on separate survey pages, were (1) the installation of an app that tracks smartphone usage behavior, (2) the linkage of administrative data (employment records), (3) the collection of biomarkers, (4) the linkage of bank account data, (5) the linkage of data from a respondent's Facebook account, (6) the linkage of health insurance data, and (7) the gathering of data measured by smartphone sensors (e.g., GPS and barometer). The exact wording of the requests can be found in Online Appendix A. For each of the seven requests, respondents could select "Yes, I agree" (coded 1) or "No, I do not agree" (coded 0).

Incentives. Respondents were randomly assigned to one of two incentives groups (no incentive vs. incentive). When answering the consent requests, respondents were only informed that they would receive an incentive or that they would not, but they were not informed about the concrete incentive amount. For our analyses, we coded the no incentive group with 0 and the incentive group with 1.

Question Wording. Respondents were randomly assigned to one of three question wording groups. Based on the experimental groups, respondents were coded with 0 if they received no 
beneficial framing, with 1 for the scientific benefit framing (emphasizing the scientific use of the data) and 2 for the time saving benefit framing (emphasizing the possibility to shorten the questionnaire by consenting).

\subsubsection{Control variables}

Gender. At the beginning of the questionnaire, we asked respondents if they were male or female. It was mandatory to answer the question since it was used for screening to meet the defined quotas. Female was coded as 1 , and male was coded as 0 .

Age. Respondents had to provide their age in years, and skipping this question was not possible.

Education. Education was also used for screening, and thus, another mandatory question that was asked in a closed-ended format. For our analysis, we recoded education into three categories from low to high, in accordance with the German school system (9-, 10-, and 12/13-year school tracks).

Device. The device variable was coded as 0 for respondents using a desktop/laptop computer to complete the survey and as 1 for respondents using a smartphone.

\subsection{Analyses}

All analyses were conducted in R version 3.6.2 (R Core Team, 2018). For data analysis, we created a dataset consisting of all respondents who gave an answer to all seven consent requests, reducing the dataset to 3,327 cases to ensure comparability across data domains. We then transformed the dataset into the long format using each consent decision as an observation that is nested within a respondent. To answer Research Questions 1 and 2, we specified a logistic multilevel model predicting the probability of consent, including data domains and experimental manipulations nested in the respondent (Model 1). These 
manipulations are question order, incentive, and question wording. The regression model allows us to estimate the main effects of the data domain and our experimental manipulations on linkage consent. To answer Research Question 3, we added interactions between the data domains and the experimental manipulations to our model (Model 2). This allows us to identify differences in the effects caused by a manipulation based on the data domain. In both models, control for device, gender, and education. ${ }^{4}$ To estimate the multilevel regression models, we used the lme4 library (Bates et al., 2015).

\section{Results}

\subsection{Does consent rates for data linkage differ by data domain?}

In Model 1 (see Table B.1 in the Appendix), where administrative data is the reference category, we see that consent rates significantly differ for app data $(p=.01)$, biomarker $(p<$ $.001)$, Facebook $(p=.002)$, and bank $(p<.001)$ data, thus supporting H1. Figure 2 displays the predicted probabilities of consent by data domain. Except for the linkage of bank account data (19\%), 95\% CI [14.1, 24.4], all consent probabilities ranged between $45 \%$ for sensor data, 95\% CI [39.0, 55.4] and 54\% for biomarkers, 95\% CI [46.0, 62.0]. It should be noted that predicted probabilities do not resemble regression coefficients but result out of a specific configuration of the regression model where reference categories are held constant for the calculation. Thus, significant differences as displayed in Table B.1 might not be visible in the graph.

[Figure 2 about here.]

\footnotetext{
${ }^{4}$ We controlled for gender and education but not for age because the former two variables were not equally distributed between the incentive conditions. The device manipulation needed to be controlled for as it caused respondents to break-off the survey, thus creating a response bias.
} 


\subsection{How do contextual factors (i.e., question order, consent question wording, and incentives) influence consent rates?}

To answer Research Question 2, we again use Model 1, including main effects only. Our regression model shows significant effects comparing Position 1 to all other positions, thus supporting H2.1. As shown by the predicted probabilities of consent displayed in Figure 3, the consent probability drops when a question is asked in the second (22\%, 95\% CI [16.6\%, $28.2 \%])$ instead of the first position $(46 \%, 95 \%$ CI $[38.0 \%, 54.5 \%])$. This trend continuous throughout all seven positions with a declining strength. Eventually, consent probabilities stay nearly consistent between the fourth (X\%, 95\% CI $[7.3 \%, 13.4 \%])$ and the seventh position $(\mathrm{X} \%, 95 \% \mathrm{CI}[4.5 \%, 8.5 \%])$.

[Figure 3 about here.]

Concerning the influence of consent question wording, we do not find a significant effect for any of the variations ( $\mathrm{p}>.1$, see Table B.1). Therefore, H2.2 is not supported. Similarly, the incentive condition did not significantly influence the consent probability ( $p>.1$, see Table B.1), leading to a lack of support for $\mathrm{H} 2.3$.

\subsection{How do data domains and contextual factors interact with respect to willingness to share additional data?}

To examine how the effect of our experimental manipulations differ by data domains, we specified a multilevel logistic regression model including interaction terms between domains and manipulations (Model 2 in the Online Appendix Table B.2).

When looking at the interaction between data domain and question order, we find several significant effects. Figure 3 shows the predicted probabilities of consent by question order and data domain. All seven data domains show a common pattern of the probability of consent continuously declining between the first and seventh positions. However, for some 
domains, the effect between the first and the second position is more pronounced (administrative data, bank data, biomarkers, Facebook data, health insurance data) than for others (app data, sensor data), where the 95\% confidence intervals overlap.

[Figure 4 about here.]

None of the interactions between data domain and question wording are significant ( $\mathrm{p}>.1$, see Table B2). Concerning the interaction between data domain and incentive, we find significant interactions between Facebook data and incentive group $(p=.014$, see Figure 5 and Table B.2) and between app data and incentive ( $p=.031$, see Figure 5 and Table B.2).

[Figure 5 about here.]

\section{Discussion}

In this paper, we investigated respondents' willingness to consent to data linkage for seven different data domains in a web survey. We found variation in the likelihood to consent depending on the data domain and the position at which the data linking request was asked. Specifically, asking for consent to link app data, biomarker data, and Facebook data to the survey responses created higher consent rates (all larger than 50\% when in first position) compared to administrative data (63.0\% when in first position), which did overall not significantly differ from consent likelihood to health insurance (46.5\% when in first position) and sensor data (46.3\% when in first position). By far the lowest likelihood to consent was observed for bank account data (33.8\% when in first position). This finding is in line with research on the sensitivity of certain survey topics (Singh \& Hill, 2003; Tourangeau \& Yan, 2007), e.g., that financial information, in general, is considered to be more private and sensitive than information about other data domains.

Furthermore, the results showed that the likelihood to consent to data linkage is negatively associated with the question position. All data domains achieved significantly 
higher consent rates when the request came first in the sequence compared to when the same question was asked at a later position. Our result is in line with the findings by Keusch et al. (2019). The likelihood to consent further decreased with each additional request, showing that respondents may have the feeling of increasing privacy costs with every additional linkage of data they allow. This might be caused by the feeling that researchers can get a more complete picture of the respondent with every additional data domain linked. Interestingly, the decline in the consent rate with every additional request was not consistent across data domains, with some domains showing a steeper decline from the first to the second request than others. Together with findings from Walzenbach et al. (2021), we see a first indication that starting with a less sensitive data domain might be advantageous.

Besides the question order, we also investigated the influence of the consent question wording and promising an incentive on the likelihood to consent to data linkage. When considering the effect of consent question wording, we found that neither outlining the scientific benefit of the data linkage nor the time savings for respondents increased the consent rate compared to not providing any beneficial argument. We also found no interaction between question wording and data domain. Similar findings were reported by Sakshaug et al. (2013) and Pascale (2011), while other research found variations of question wording to increase consent rates (Kreuter et al., 2015; Sakshaug \& Kreuter, 2014). A possible explanation for this null effect could have been the separation of stimulus and question in our study, as the beneficial phrase was placed on the introductory page of the consent module before the specific consent request(s) and not on the same page as the request(s). Additionally, we found no effect of an incentive, contradicting our assumptions. A similar null finding was reported by Jäckle et al. (2017), while Keusch et al. (2019) reported a positive effect of providing an incentive. Similar to the null effect of question wording, we assume that the separation between stimulus and consent question could have weakened the 
effect of the incentive. Additionally, the information about the incentive might not have been specific enough, as we did not state the exact amount that participants would receive upon data linkage, which may have further reduced its effect. However, while the main effect of the incentive was non-significant, we found significant interactions between the incentive treatment and data domain. The willingness to consent to the linkage of Facebook data and app data was positively affected by the incentive.

Our study is not without limitations. First, we conducted our experiments with members of a non-probability online access panel. Even though quotas for sociodemographic characteristics were used, we do have to acknowledge that our respondents may differ from the general population in Germany in other characteristics, including their likelihood to consent to data linkage requests. While we might overestimate general consent rates, because members of a nonprobability online panel who have volunteered to regularly respond to surveys and are thus used to share a lot of information might be more willing to consent to a data linkage request, our goal was not to produce population estimates with this study. In contrast, we were mainly interested in identifying casual relationships through our experimental variations, and we assume that the effects of data domain and question order we found here may also hold for the general population. Second, the questionnaire we used was about 30 minutes long, with the consent and data linkage part being located toward the end of the questionnaire. This could have resulted in an underestimation of respondents' willingness to consent and may have had a negative effect on their response behavior in general due to respondents' fatigue (Sakshaug et al., 2013).

Nevertheless, we think that our study provides valuable insights for researchers who want to implement data linkage requests in a web survey. Understanding how respondents react to different consent requests and how different factors such as the data domain or question wording can affect consent decisions will help researchers to design the consent process in a 
way that maximizes consent rates. Our study is one of the first to compare consent to data linkage requests for a variety of different data domains. Considering our results, we recommend confronting respondents only with a single consent request or, if necessary, to sort multiple consent request by sensitivity and/or importance for the researcher, starting with the ones that are least sensitive and/or in which the researcher is most interested. We further recommend, to continue the research on the effect of specific question wording and incentives, but to put this information closer to the consent request, as this design improvement will likely increase the effectivity compared to our study. 


\section{Literature}

Avendano, M., Scherpenzeel, A., \& Mackenbach, J. P. (2011). Can biomarkers be collected in an Internet survey? A pilot study in the LISS panel. Social Research and the Internet: Advances in Applied Methods and New Research Strategies, 371-412. Routledge

Baker, R., Shiels, C., Stevenson, K., Fraser, R., \& Stone, M. (2000). What proportion of patients refuse consent to data collection from their records for research purposes? British Journal of General Practice, 50(457), 655-656.

Bates, D., Mächler, M., Bolker, B., \& Walker, S. (2015). Fitting Linear Mixed-Effects Models Using lme4. Journal of Statistical Software, 67(1).

Christoph, B., Müller, G., Gebhardt, D., Wenzig, C., Trappmann, M., Achatz, J., Tisch, A., Gayer, C., \& others. (2008). Codebook and documentation of the panel study' Labour Market and Social Security'(PASS): Vol. 1: Introduction and overview, wave 1 (2006/2007). Institut für Arbeitsmarkt-und Berufsforschung (IAB), Nürnberg

Jäckle, A., Burton, J., Couper, M. P., \& Lessof, C. (2017). Participation in a mobile app survey to collect expenditure data as part of a large-scale probability household panel: Response rates and response biases. Institute for Social and Economic Research, University of Essex: Understanding Society Working Paper Series No, 09.

Keusch, F., Struminskaya, B., Antoun, C., Couper, M. P., \& Kreuter, F. (2019). Willingness to participate in passive mobile data collection. Public Opinion Quarterly, 83(S1), $210-235$.

Kreuter, F., Sakshaug, J. W., \& Tourangeau, R. (2015). The framing of the record linkage consent question. International Journal of Public Opinion Research, 28(1), 142-152.

Link, M. W., Murphy, J., Schober, M. F., Buskirk, T. D., Hunter Childs, J., \& Langer Tesfaye, C. (2014). Mobile technologies for conducting, augmenting and potentially replacing surveys: Executive summary of the AAPOR task force on emerging 
technologies in public opinion research. Public Opinion Quarterly, 78(4), 779-787.

Martin, K., \& Shilton, K. (2016). Putting mobile application privacy in context: An empirical study of user privacy expectations for mobile devices. The Information Society, 32(3), 200-216.

McFall, S. L., Conolly, A., \& Burton, J. (2014). Collecting biomarkers using trained interviewers. Lessons learned from a pilot study. Survey Research Methods, 8, 57-66.

Nissenbaum, H. (2018). Respecting Context to Protect Privacy: Why Meaning Matters. Science and Engineering Ethics, 24(3), 831-852.

Nissenbaum, H. (2019). Contextual Integrity Up and Down the Data Food Chain. Theoretical Inquiries in Law, 20(1), 221-256.

Pascale, J. (2011). Requesting Consent to Link Survey Data to Administrative Records: Results from a Split-Ballot Experiment in the Survey of Health Insurance and Program Participation (SHIPP). Survey Methodology, 03.

R Core Team. (2018). R: A Language and Environment for Statistical Computing. R Foundation for Statistical Computing. https://www.R-project.org/

Revilla, M., Couper, M. P., \& Ochoa, C. (2018). Willingness of online panelists to perform additional tasks. Methods, Data, Analyses, 13(2), 29.

Sakshaug, J., Tutz, V., \& Kreuter, F. (2013). Placement, wording, and interviewers: Identifying correlates of consent to link survey and administrative data. Survey Research Methods, 7, 133-144.

Sakshaug, J. W., Couper, M. P., Ofstedal, M. B., \& Weir, D. R. (2012). Linking survey and administrative records: Mechanisms of consent. Sociological Methods \& Research, 41(4), 535-569.

Sakshaug, J. W., \& Kreuter, F. (2014). The effect of benefit wording on consent to link survey and administrative records in a web survey. Public Opinion Quarterly, 78(1), 
$166-176$.

Sakshaug, J. W., Schmucker, A., Kreuter, F., Couper, M. P., \& Singer, E. (2019). The effect of framing and placement on linkage consent. Public Opinion Quarterly, 83(S1), 289308.

Singer, E., \& Ye, C. (2013). The use and effects of incentives in surveys. The ANNALS of the American Academy of Political and Social Science, 645(1), 112-141.

Silber, H., Breuer, J., Beuthner, C., Gummer, T., Keusch, F., Siegers, P., Stier, S., \& Weiß, B. (2021). Linking surveys and digital trace data: Insights from two studies on determinants of data sharing behavior [Preprint]. SocArXiv.

Singh, T., \& Hill, M. E. (2003). Consumer privacy and the Internet in Europe: A view from Germany. Journal of Consumer Marketing.

Struminskaya, B., Toepoel, V., Lugtig, P., Haan, M., Luiten, A., \& Schouten, B. (2021). Understanding willingness to share smartphone-sensor data. Public Opinion Quarterly, 84(3), 725-759.

Tourangeau, R., \& Yan, T. (2007). Sensitive questions in surveys. Psychological Bulletin, $133(5), 859$.

Walzenbach, S., Burton, J., Couper, M. P., Crossley, T. F., Annette, J., \& others. (2021). Experiments on multiple requests for consent to data linkage in surveys. Understanding Society at the Institute for Social and Economic Research.

Wang, L., Zhang, Z., McArdle, J. J., \& Salthouse, T. A. (2008). Investigating Ceiling Effects in Longitudinal Data Analysis. Multivariate Behavioral Research, 43(3), 476-496.

Wenz, A., Jackle, A., \& Couper, M. P. (2019). Willingness to use mobile technologies for data collection in a probability household panel. Survey Research Methods, 13(1), 122. 
Introductory text with beneficial statement and information regarding data linkage and data security

\section{P 5}

Different data linkage requests

(App, Administrative Data, Bank Account, Biomarker, Facebook, Health Insurance, Sensor Data)

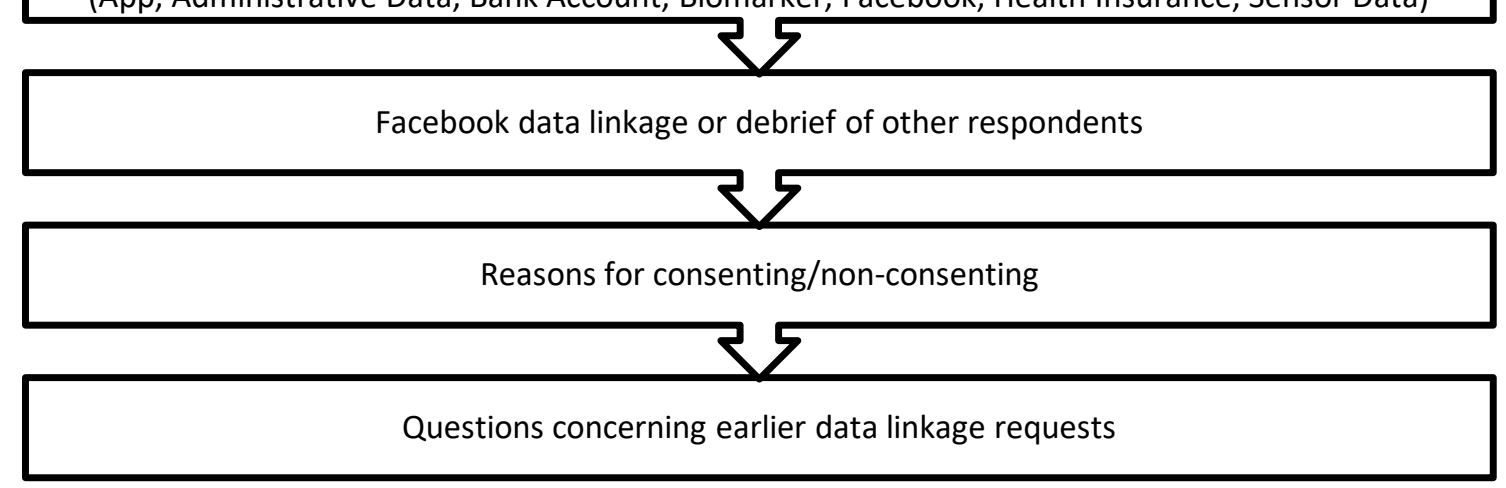

Figure 1: Steps of the Consent for Data Linkage Module 


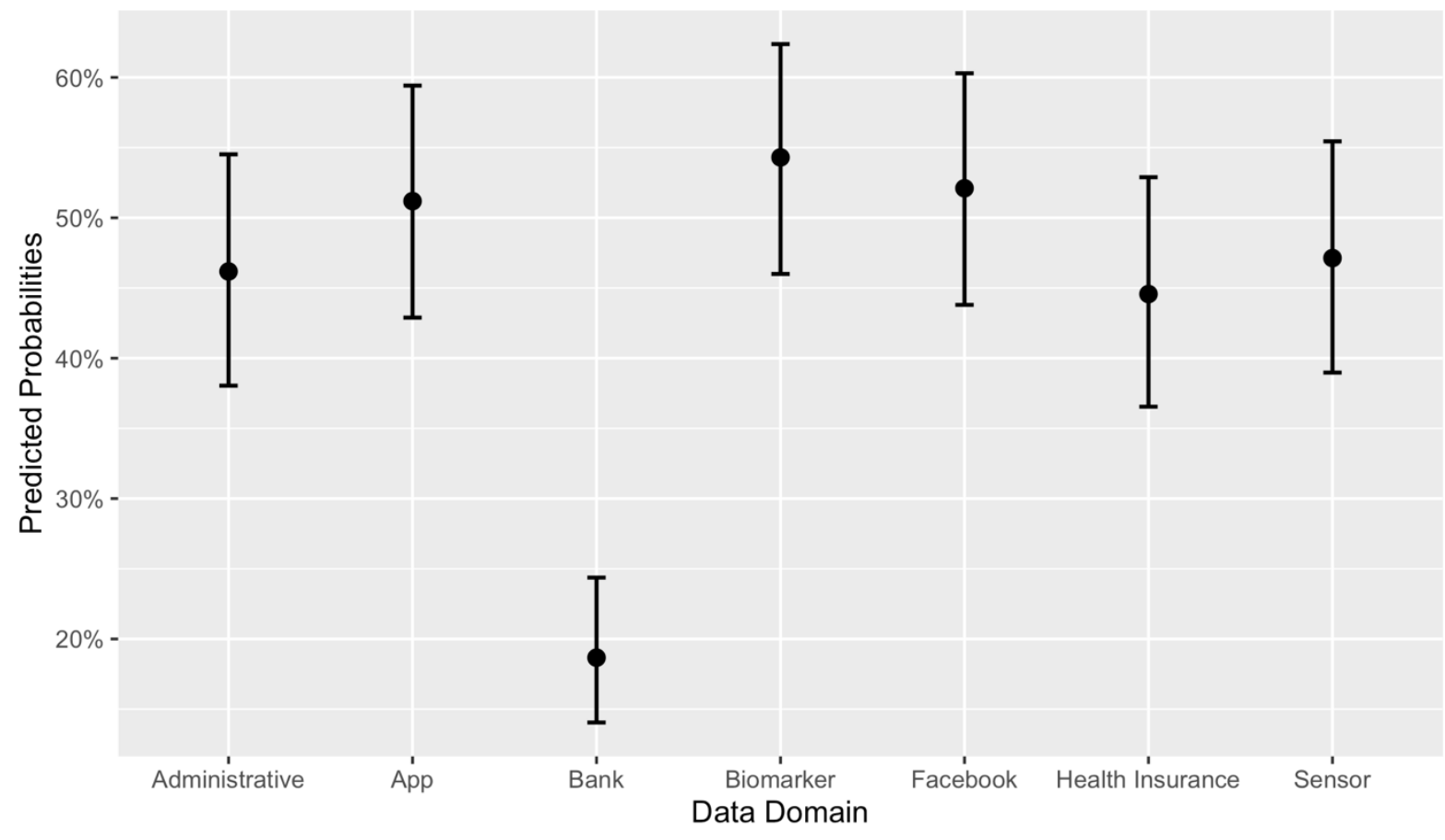

Figure 2. Predicted Probabilities by Data Domain (see Model 1 in Table B.1) 


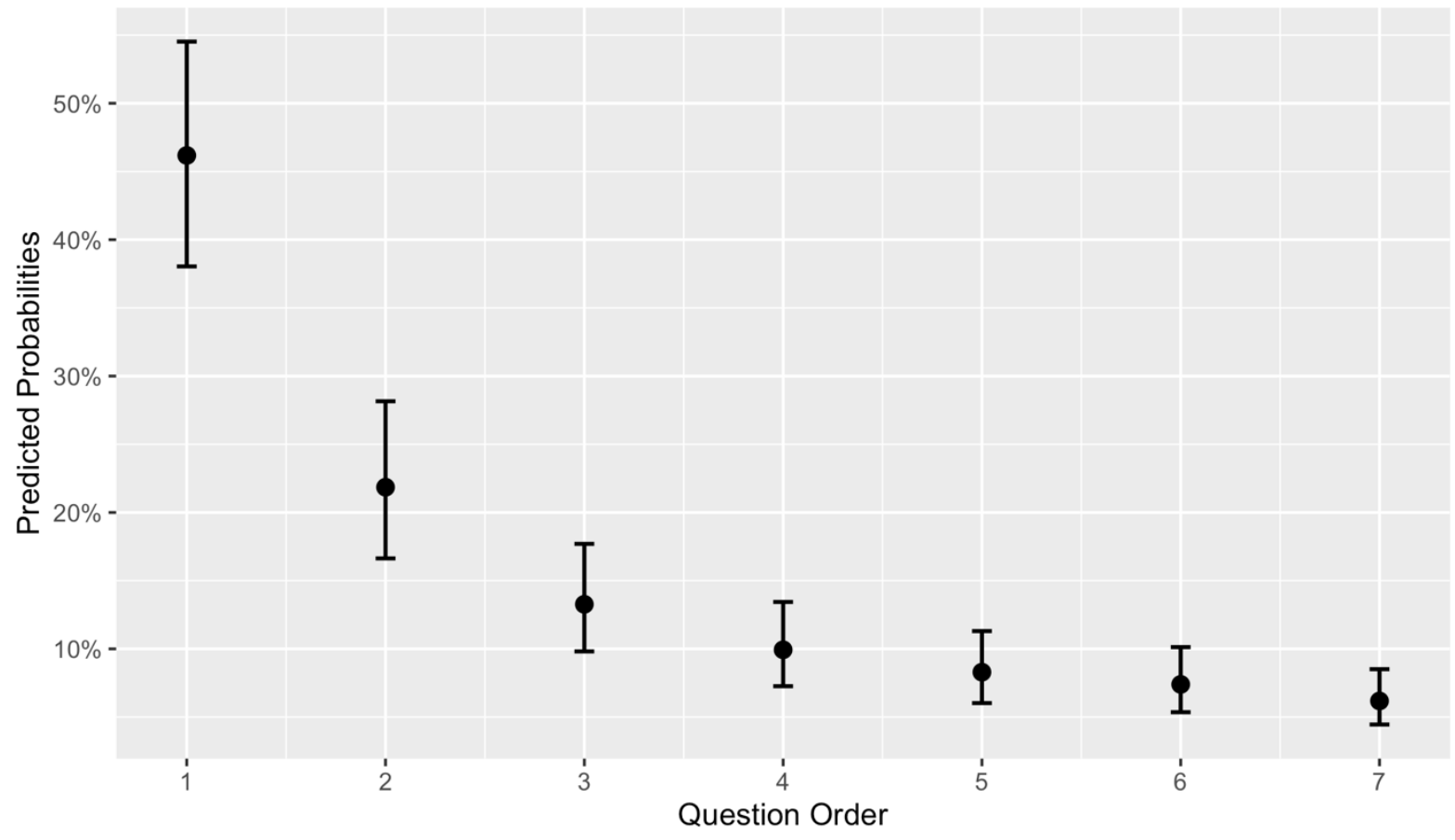

Figure 3. Predicted probabilities of consent to data domain by consent question position (see Model 1 in Table B.1) 


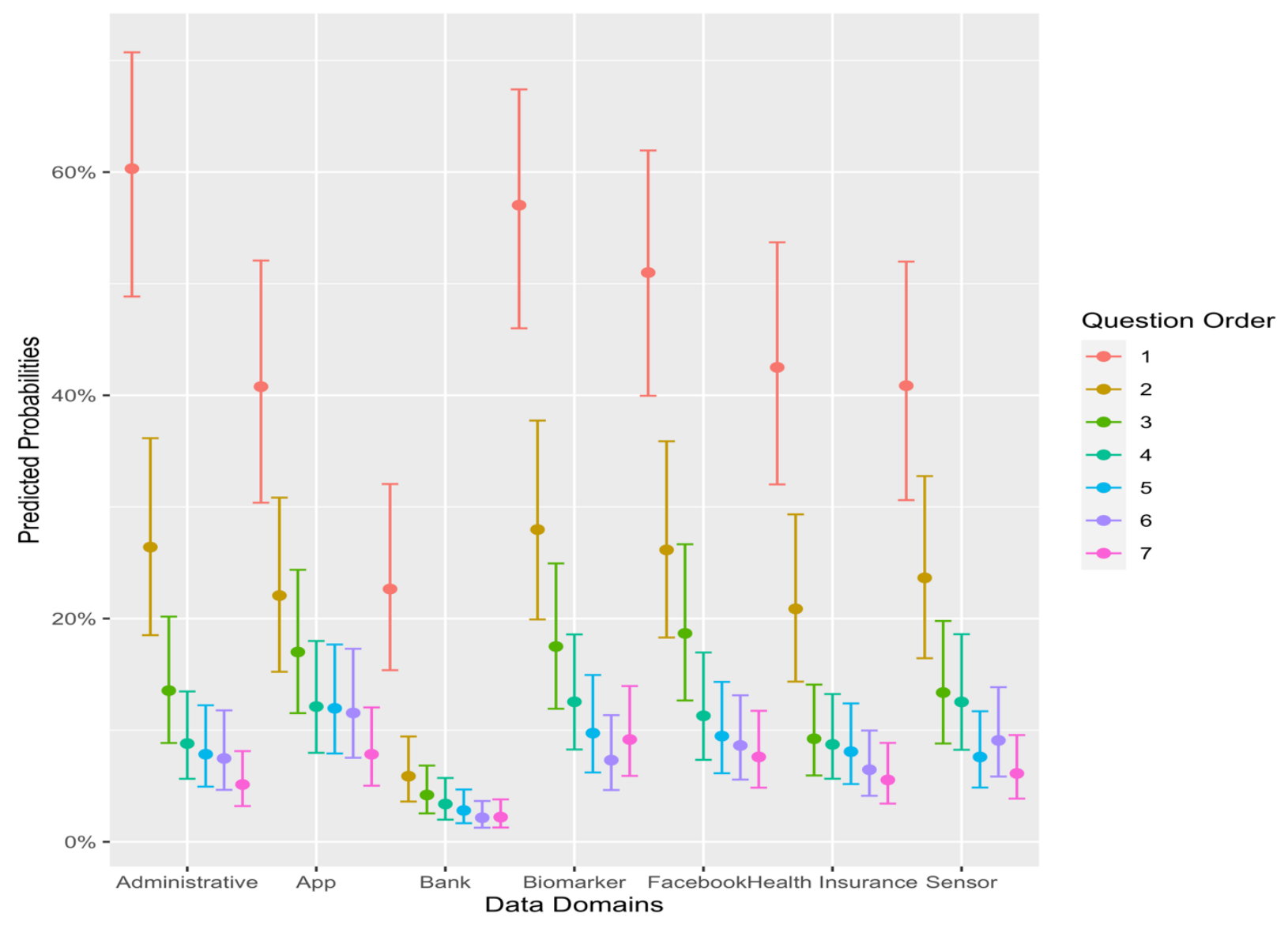

Figure 4. Predicted probabilities between Data Domain and Question Order (see Model 2 in Table B.2) 


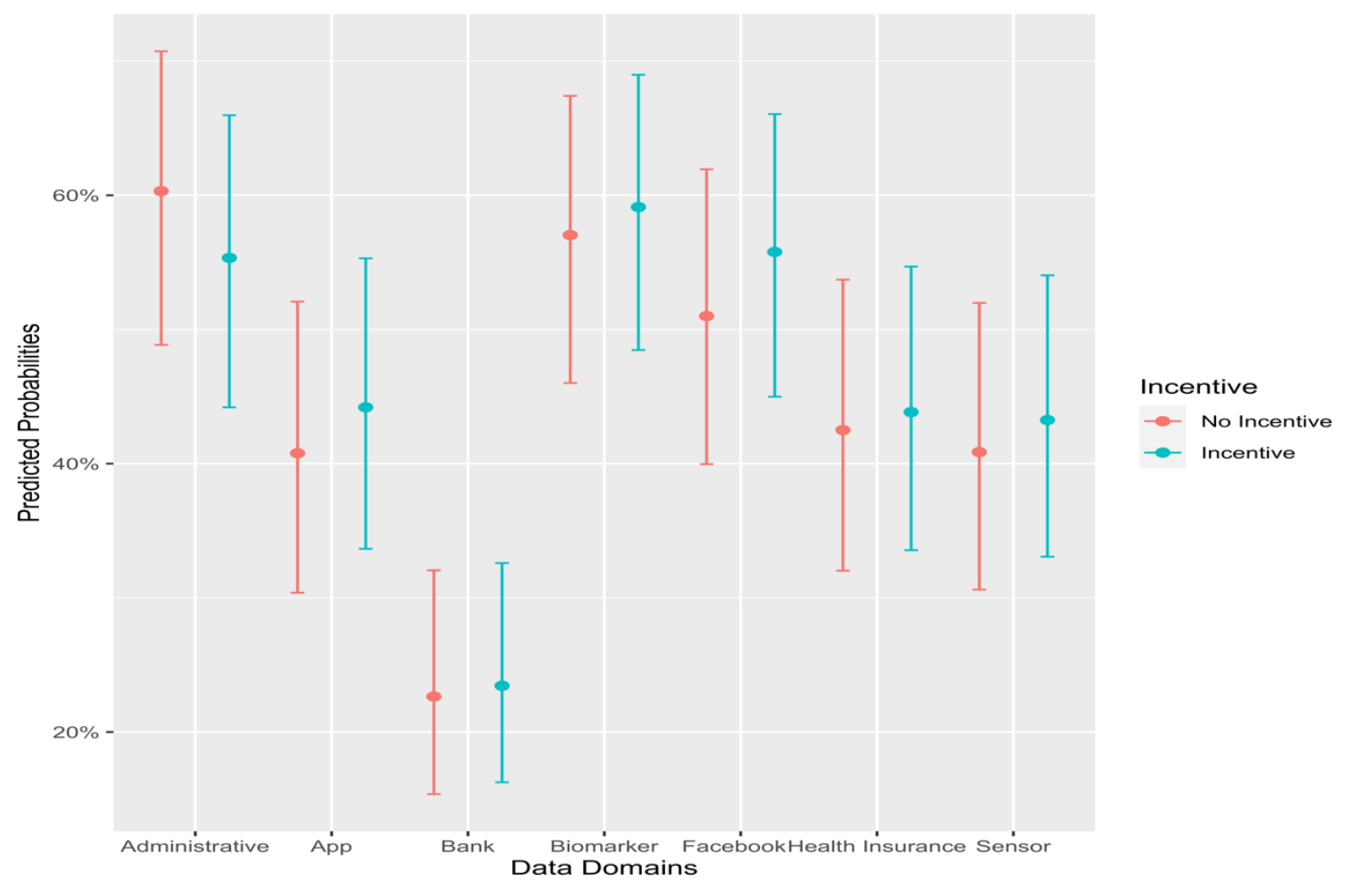

Figure 5. Predicted probabilities between Data Domain and Incentive (see Model 2 in Table B.2) 
Table 1. Descriptive Statistics

\begin{tabular}{|c|c|c|c|c|c|}
\hline \multicolumn{5}{|l|}{ Variable } & \multirow{2}{*}{$\begin{array}{l}\text { Missing } \\
\text { Values }\end{array}$} \\
\hline & & & & & \\
\hline \multirow[t]{3}{*}{ Age } & Range & Median & Mean & Standard & \\
\hline & & & & Deviation & \\
\hline & $18-70$ & 44 & 43.8 & 13.9 & 16 \\
\hline \multirow[t]{3}{*}{ Education } & & Low Education & Medium & High & \\
\hline & & & Education & Education & \\
\hline & & $14.3 \%$ & $41.7 \%$ & $44.0 \%$ & 0 \\
\hline \multirow[t]{2}{*}{ Gender } & & Male & Female & & \\
\hline & & $42.8 \%$ & $57.2 \%$ & & 16 \\
\hline \multirow[t]{2}{*}{ Incentives } & & No Incentive & Incentive & & \\
\hline & & $41.8 \%$ & $58.2 \%$ & & 0 \\
\hline Question & & Neutral & Scientific & Time Saving & \\
\hline \multirow[t]{2}{*}{ Wording } & & & Benefit & & \\
\hline & & $33.5 \%$ & $33.0 \%$ & $33.5 \%$ & 0 \\
\hline \multirow[t]{2}{*}{ Device } & & Desktop/Laptop & Smartphone & & \\
\hline & & $53.7 \%$ & $46.3 \%$ & & 0 \\
\hline
\end{tabular}

Note. $\mathrm{n}=3,327$ 


\section{Online Appendix A: Questionnaire Wording}

Introductory Text

[In addition to your responses/ For scientific purposes/ In order to shorten the survey duration and save you some questions,] we would like to collect some data in addition to your answers that are of interest for our evaluation. In this way, you support our research and make a valuable contribution to scientific progress.

In order to combine these data with your survey data, we will ask for your consent in each case. When evaluating the data, we absolutely ensure that all data protection regulations are complied with and that the data are not passed on to third parties.

Your consent is, of course, voluntary. You can revoke it at any time at onlinestudie2018@gesis.org.

In the following, you will be asked in each case for your consent to the use of this data. You will then be randomly selected for certain data and will receive detailed information on the further course of the study. [As compensation for the additional effort, you will receive additional mingle points from us.] 
Consent Requests

Administrative Data

We would like to collect some data which we can obtain from the competent authorities.

These include, for example, information on previous employment relationships, periods of unemployment and participation in measures during unemployment, and characteristics of your employer. We use these data to explore the increasing complexity of work in our society.

Do you agree?

[ ] Yes, I agree

[ ] No, I don't agree.

\section{App Data}

We would like to collect some data that we collect with a program (an "app") on your smartphone. For example, we may collect information about the frequency of smartphone use, the number of apps used, or other aspects of usage behavior. With the help of this data, we investigate human behavior in an increasingly digitalized world.

Do you agree?

[ ] Yes, I agree

[ ] No, I don't agree.

Bank Data

We would like to collect some data that we request from your bank with your consent. This includes, for example, information on consumption and savings behavior as well as income levels. With the help of this data, we investigate consumption and saving behavior.

Do you agree? 
[ ] Yes, I agree

[ ] No, I don't agree.

\section{Biomarker}

We would like to collect some data, which we determine with the help of saliva and blood samples. This includes, for example, information on environmental pollution as well as data on hormonal values. We use these data to investigate the relationship between environmental conditions and health status.

Do you agree?

[ ] Yes, I agree

[ ] No, I don't agree.

\section{Facebook Data}

We would like to collect some data from your Facebook account. This includes key data such as the number of friends or the number of posts on your own or on other people's walls. With the help of this data, we investigate the changes in interaction through digital media and the Internet.

Do you agree?

[ ] Yes, I agree

[ ] No, I don't agree.

Health Insurance Data

We would like to collect some data, which we request from your health insurance company with your consent. This includes, for example, information such as prescribed medication, 
examinations performed or the frequency of visits to the doctor. With the help of this data, we investigate differences between statutory and private health insurance companies.

Do you agree?

[ ] Yes, I agree

[ ] No, I don't agree.

Sensor Data

We would like to collect some data that using the sensors on your smartphone. These include GPS location data, ambient brightness information, and air pressure measurements. With the help of these data we investigate the influence of your environment on your behavior.

Do you agree?

[ ] Yes, I agree

[ ] No, I don't agree. 
Online Appendix B: Supplementary Tables

Table B.1. Estimates and standard errors (in parentheses) from multilevel regression predicting consent to data linkage (Model 1)

\section{H1: Data Domain (Reference:}

Administrative Data)

$\begin{array}{llc}\text { App Data } & 0.201^{* * * *} & (0.078) \\ \text { Bank Data } & -1.319^{* * * *} & (0.086) \\ \text { Biomarker Data } & 0.326^{* * *} & (0.077) \\ \text { Facebook Data } & 0.237^{* * *} & (0.078) \\ \text { Health Insurance Data } & -0.065 & (0.079) \\ \text { Sensor Data } & 0.038 & (0.078)\end{array}$

H2.1: Question Position:

(Reference: Position 1)

$\begin{array}{lll}\text { Position } 2 & -1.121^{* * *} & (0.072) \\ \text { Position } 3 & -1.724^{* * *} & (0.075) \\ \text { Position } 4 & -2.052^{* * *} & (0.077) \\ \text { Position } 5 & -2.250^{* * * *} & (0.079) \\ \text { Position } 6 & -2.374^{* * *} & (0.080) \\ \text { Position } 7 & -2.567^{* * *} & (0.081)\end{array}$

H2.2: Question Wording

(Reference: No Benefit) 
Science Benefit

0.081

$(0.141)$

Time Benefit

$-0.078$

$(0.141)$

\section{H2.3: Incentive (Reference: No}

\section{Incentive)}

$$
0.058
$$

Female

$$
0.741^{* * *}
$$

Education (Reference: High)
Medium
$0.234^{*}$
(0.126)
Low
$0.416^{* *}$
(0.176)

Device Mobile (Reference:

$-0.228^{*}$

(0.117)

Desktop/Laptop)

Constant

$-0.153$

$(0.171)$

Observations

23,177

Log Likelihood

$-9,589.202$

Akaike Inf. Crit.

$19,220.400$

Bayesian Inf. Crit.

$19,389.470$

${ }^{*} \mathrm{p}<0.05^{* *} \mathrm{p}<0.01^{* * *} \mathrm{p}<0.001$ 
Table B.2. Estimates and standard errors (in parentheses) from multilevel regression predicting consent to data linkage including interactions (Model 2)

H1: Data Domain (Reference:

Administrative Data)

$\begin{array}{llc}\text { App Data } & -0.791^{* * * *} & (0.257) \\ \text { Bank Data } & -1.647^{* * * *} & (0.265) \\ \text { Biomarker Data } & -0.135 & (0.252) \\ \text { Facebook Data } & -0.378 & (0.252) \\ \text { Health Insurance Data } & -0.720^{* * *} & (0.255) \\ \text { Sensor Data } & -0.788^{* * * *} & (0.254)\end{array}$

H2.1: Question Position (Reference:

\section{Position 1)}

Position 2

Position 3

Position 4

Position 5

Position 6

Position 7
$-1.443^{* * *}$

$-2.272^{* * *}$

$-2.756^{* * * *}$

$-2.882^{* * *}$

$-2.935^{* * *}$

$-3.335^{* * *}$
(0.211)

\section{H2.2: Question Wording (Reference:}

\section{No Benefit)}

Science Benefit

0.134

Time Benefit

0.086

(0.193) 
H2.3: Incentive (Reference: No

$(0.160)$

Incentive)

$-0.204$

Female

$0.739^{* * *}$

Education (Reference: High)

Medium

Low

Device Mobile (Reference:

Desktop/Laptop)

\section{Interaction Effect: Data Domain *}

\section{Question Position}

$\begin{array}{lc}\text { App Data * Position } 2 & 0.554^{*} \\ \text { Bank Data * Position 2 } & -0.101 \\ \text { Biomarker Data * Position 2 } & 0.214 \\ \text { Facebook Data * Position 2 } & 0.365 \\ \text { Health Insurance Data * Position 2 } & 0.413 \\ \text { Sensor Data * Position 2 } & 0.641^{* *} \\ \text { App Data * Position 3 } & 1.060^{* * *} \\ \text { Bank Data * Position 3 } & 0.372 \\ \text { Biomarker Data * Position 3 } & 0.438 \\ \text { Facebook Data * Position 3 } & 0.760^{* *} \\ \text { Health Insurance Data * Position 3 } & 0.289 \\ \text { Sensor Data * Position 3 } & 0.773^{* *} \\ \text { App Data * Position } 4 & 1.148^{* * *}\end{array}$

$-0.235^{* *}$ 


\begin{tabular}{|c|c|c|}
\hline Bank Data * Position 4 & $0.634^{*}$ & $(0.335)$ \\
\hline Biomarker Data $*$ Position 4 & $0.531^{*}$ & $(0.310)$ \\
\hline Facebook Data * Position 4 & $0.655^{* *}$ & $(0.311)$ \\
\hline Health Insurance Data * Position 4 & $0.711^{* *}$ & $(0.311)$ \\
\hline Sensor Data * Position 4 & $1.183^{* * * *}$ & $(0.309)$ \\
\hline App Data $*$ Position 5 & $1.259^{* * * *}$ & $(0.319)$ \\
\hline Bank Data * Position 5 & $0.566^{*}$ & $(0.338)$ \\
\hline Biomarker Data $*$ Position 5 & 0.372 & $(0.323)$ \\
\hline Facebook Data * Position 5 & $0.584^{*}$ & $(0.317)$ \\
\hline Health Insurance Data $*$ Position 5 & $0.752^{* *}$ & $(0.319)$ \\
\hline Sensor Data $*$ Position 5 & $0.753^{* *}$ & $(0.321)$ \\
\hline App Data * Position 6 & $1.272^{* * *}$ & $(0.326)$ \\
\hline Bank Data * Position 6 & 0.349 & $(0.341)$ \\
\hline Biomarker Data $*$ Position 6 & 0.112 & $(0.325)$ \\
\hline Facebook Data * Position 6 & $0.535^{*}$ & $(0.320)$ \\
\hline Health Insurance * Position 6 & $0.564^{*}$ & $(0.322)$ \\
\hline Sensor Data * Position 6 & $1.001^{* * *}$ & $(0.321)$ \\
\hline App Data $*$ Position 7 & $1.244^{* * *}$ & $(0.323)$ \\
\hline Bank Data * Position 7 & $0.775^{* *}$ & $(0.343)$ \\
\hline Biomarker Data $*$ Position 7 & $0.758^{* *}$ & $(0.322)$ \\
\hline Facebook Data * Position 7 & $0.798^{* *}$ & $(0.321)$ \\
\hline Health Insurance Data $*$ Position 7 & $0.802^{* *}$ & $(0.330)$ \\
\hline
\end{tabular}


Sensor Data * Position 7

$0.975^{* * *}$

$(0.323)$

\section{Interaction Effect: Data Domain *}

\section{Question Wording}

$\begin{array}{lll}\text { App Data * Science Benefit } & -0.094 & (0.191) \\ \text { Bank Data * Science Benefit } & -0.354^{*} & (0.214) \\ \text { Biomarker Data * Science Benefit } & 0.047 & (0.192) \\ \text { Facebook Data * Science Benefit } & -0.137 & (0.194) \\ \text { Health Insurance * Science Benefit } & 0.178 & (0.193) \\ \text { Sensor Data * Science Benefit } & -0.115 & (0.193) \\ \text { App Data * Time Benefit } & -0.242 & (0.213) \\ \text { Bank Data * Time Benefit } & -0.156 & (0.194) \\ \text { Biomarker Data * Time Benefit } & -0.177 & (0.195) \\ \text { Facebook Data * Time Benefit } & -0.238 & (0.197) \\ \text { Health Insurance Data * Time Benefit } & -0.118 & (0.195) \\ \text { Sensor Data * Time Benefit } & -0.143 & \end{array}$

\section{Interaction Effect: Data Domain*}

\section{Incentive}

$\begin{array}{llc}\text { App Data * Incentive } & 0.344^{* *} & (0.160) \\ \text { Bank Data * Incentive } & 0.249 & (0.177) \\ \text { Biomarker Data * Incentive } & 0.290^{*} & (0.160) \\ \text { Facebook Data * Incentive } & 0.396^{* *} & (0.161) \\ \text { Health Insurance Data * Incentive } & 0.259 & (0.162) \\ \text { Sensor Data * Incentive } & 0.302^{*} & (0.161)\end{array}$


Constant

$0.418^{*}$

$(0.237)$

Observations

23,177

Log Likelihood

$-9,546.980$

Akaike Inf. Crit.

$19,243.960$

Bayesian Inf. Crit.

$19,847.780$

${ }^{*} \mathrm{p}<0.05^{* *} \mathrm{p}<0.01^{* * *} \mathrm{p}<0.001$ 